\title{
Machine Vision Systems for Plant Growth Measurement and Modeling
}

\author{
Ta-Te Lin, Chung-Fang Chien, Wen-Chi LiaO, Kuo-Chi Chung and Jen-Min Chang \\ Department of Bio-Industrial Mechatronics Engineering, National Taiwan University, \\ Taipei 106, Taiwan, ROC
}

(Received July 1, 2006)

\begin{abstract}
Recent developments of microcomputer-based machine vision systems has offered convenient and non-destructive ways for measurements of plant characteristics that allow plant growth assessment. This paper presents brief reviews and comparisons of machine vision approaches for non-destructive plant growth measurement. The simple approach of using projected silhouette image of a plant was most commonly used in acquiring growth data in various experiments. Plant fresh or dry weight can be indirectly estimated from projected leaf area by calibration with data from standard measurement methods. However, the estimation error usually increases as the extent of overlapped leaves increases. Images acquired from different views or utilizing dual cameras allow the estimation of leaf area without predetermined calibration relationship but the image processing algorithms usually become more sophisticated. By incorporating multiple images of a plant, three-dimensional and structural information may be extracted for more detailed growth analyses and modeling.
\end{abstract}

Keywords : image processing, Hough transform, leaf area, non-destructive measurement

\section{INTRODUCTION}

Plant growth measurement is important for studies of plant physiology as well as various horticultural applications. The information related to plant characteristics is especially useful for its applications in weed and pest control, crop modeling and climate control in greenhouses, etc. To efficiently acquire plant features, several approaches have been developed. Many methods to measure leaf area using optical devices or photoelectric meters were reported (Robinson et al., 1970; Daughtry, 1990). Other approaches to evaluate plant growth by measuring leaf dimensions, leaf specific weight, stem diameter, leaf and petiole angles, and roots have been proposed as well (Singh and Singh, 1983; Palit and Battacharyya, 1984; Wolfe and Sandler, 1985). Recent development of microcomputer-based image processing systems has further boosted the applications of machine vision techniques for plant characteristics assessment (Howarth and Stanwood, 1993; Nyakwende et al., 1997). To elucidate both hardware and software fundamentals of designing a machine vision system for plant feature measurement, this paper briefly reviews the methods of related researches and focuses on the comparisons of two machine vision approaches, projected silhouette and structural approaches, for plant feature extraction, measurement, and modeling.

Many successful applications have been reported in plant growth monitoring and plant shape

Corresponding author: Ta-Te Lin, fax : 886-2-23929416, e-mail : m456@ntu.edu.tw 


\section{T. T. LIN ET AL.}

recognition using machine vision technique. Meyer and Davison (1987) demonstrated that video imagery methods worked well for determining in situ leaf area, stem diameter, and leaf and petiole angles of soybeans. Suh and Miles (1988) successfully applied a machine vision system to measure morphological characteristics of tree seedlings. Hack (1989) developed an image processing system for leaf area measurement of lettuce in hydroponic culture. A similar approach was also successfully applied in the growth measurement and modeling of lettuce based on the calculated relative soil coverage (Henten and Bontsema, 1995).

In determining leaf area from the acquired image, one of the prerequisites is to segment the plant leaves from the background. Following this segmentation process, the designated plant features are usually determined from projected area by proper calibration. The calibration is based on the assumption that the projected area correlates with the plant features. In fact, the correlations between leaf area and fresh or dry plant weights are usually high (Bailey and Steward, 1982). The projection area of plant images was also reported to have high correlation with total leaf area, fresh and dry plant weights in many studies (Storlie et al., 1989; Shibata et al., 1992; Suzuki, 1995).

By utilizing special lighting setup or multiple images from different views, three-dimensional and structural information may be extracted for more detailed plant growth analyses and modeling. Trooien and Heermann (1992) applied image processing method and modeling approach to study potato canopy structure and measure leaf area. Shimizu and Heins (1995) used an infrared lighting apparatus and a vision system to conduct non-destructive, three-dimensional plant growth analyses of Verbena bonariensis plants. Ivanov et al. (1995) presented a method for building a 3D model for maize canopy based on stereovision. Two parallel cameras were used to record images taken from different viewpoints. Parallel to the advance of structural measurement techniques, many structural models for plants have been developed. These models allow for graphical simulation of static plant structure or dynamic plant development, and have become powerful tools to analyze the growth of plants (Prusinkiewicz et al., 1994; Fournier and Andrieu, 1998). For dynamic growth simulation, the determination of growth functions for a specific plant requires the measurement of time-dependent plant features. Machine vision approach provides an efficient way to continuously monitor and measure the features of the same plant in its growing process and thus facilitates the determination of parameters of growth functions.

\section{MACHINE VISION SYSTEM DEVELOPMENT}

\section{Projected silhouette approach}

Image segmentation is a necessary step in acquiring plant features from the projected silhouette of a plant. Therefore, high contrast between the plant and the background in the image is usually a prerequisite. This is frequently achieved by using a back-lit setup for controlled lighting environment. For natural lighting conditions such as images acquired in outdoor field, grey-level or color contrast between plant and soil background needs to be significant in order to avoid complicated or even unmanageable segmentation procedure in a later stage.

Hardware design. Figure 1 shows the schematic diagram of a machine vision system for plant features measurement based on the projected plant silhouette approach. Plant silhouette images from different views may be taken from each plant placed on the rotary stage. The silhouette images are first binarized with an automatic threshold algorithm and the number of pixels representing the projected area of the plant is calculated and then converted to plant characteristics such as top fresh weight, top dry weight and total leaf area with calibration equations determined from separate experiments. Some other geometric features such as plant height, span, leaf and stem node numbers, and coordinates of stem nodes and leaf endpoints can also be obtained (Lin et al., 1998).

Image processing algorithms. The performance of image segmentation procedure critically affects the accuracy of plant feature measurement. Several automatic thresholding methods 


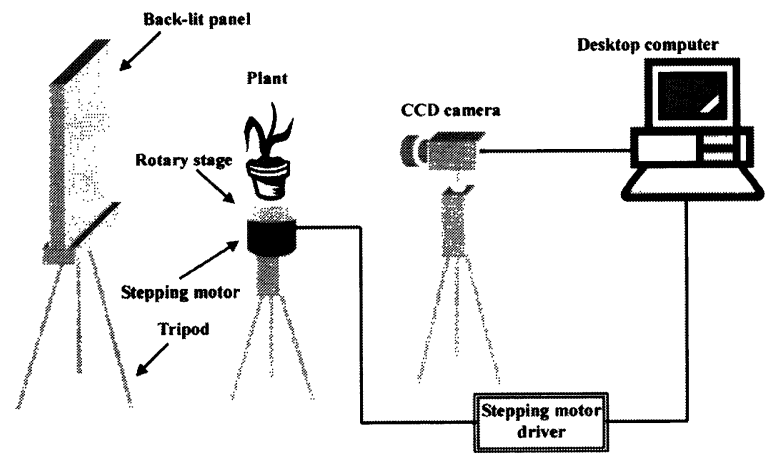

Fig. 1 Schematic diagram of the plant feature measurement system based on plant silhouette.

exist that can be implemented with minimum software programming efforts (Tsai, 1985; Gonzales and Woods, 2002). For machine vision system using color images, segmentation can be achieved by manually adjusting the threshold value for pixels expressed in various color systems such as RGB or HSI coordinates. We have developed an image segmentation algorithm based on backpropagation neural network (Lin and Chang, 1999). The segmentation process is robust for most natural lighting conditions therefore controlled lighting is not critically needed for this color machine vision system. In order to determine the plant height, leaf and node numbers and the coordinates of the nodes, the algorithm developed by Zhang and Suen (1984) was implemented to obtain the skeleton of the plant from the binary image. An algorithm was also developed to determine the nodes and plant height by tracking the connected pixels of the skeleton (Lin et al., 1998).

2. Structural approach

Hardware design. Another machine vision system based on stereo imaging was developed later (Lin and Chang, 1999). During measurement, the plant was also placed on a rotary stage controlled by a microcomputer. Stereo images of the plant were acquired from two color CCD cameras configured in perpendicular positions (Fig. 2). The machine vision system was designed to be portable to allow easy installation for automatic measurement of plant features.

Image processing algorithms. The procedure to extract plant features comprises mainly two phases: the basic setup phase and the feature extraction phase. In the basic setup phase, topview and front-view images of the plant are first acquired with dual cameras. The rotary stage then rotates 90 degrees to give the side-view images. Geometric calibration and correction are necessary for the conformation of the dimensions of the three acquired images. This step ensures the consistency of the measured features and coordinate mapping from the three images. The last procedure in the setup phase is the training and testing procedure for plant segmentation from image background using back-propagation neural network as described previously.

In order to combine information obtained from the orthogonal images, the top-view and lateral images have to be registered at the central point. The three images were acquired and calibrated in order to obtain consistent scales. Then the plant images were segmented from image background and the central point of the plant in the top-view image were determined by examining the stem position in the lateral images from their projected histograms in both horizontal and vertical directions. The stem position usually corresponded to the mode of the vertically projected histogram. The coordinates of the central point was then determined by registering the coordinates of the stem positions in the front-view and side-view images.

Following the image registration process, leaf number and axial direction of each leaf were determined from the top-view image. One developed algorithm was based on the calculation of the 


\section{T. T. LIN ET AL.}

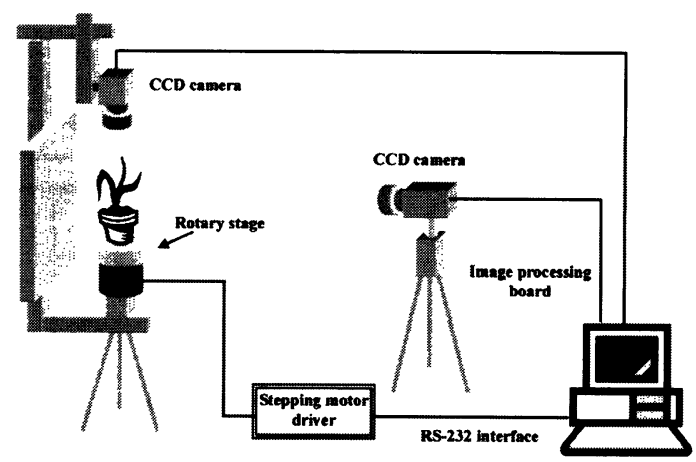

Fig. 2 Schematic diagram of the dual-camera plant feature measurement system.

distance from the leaf boundary to the central point. The tip of leaves presumably should have the maximum distance from the stem (central point). The distance from the leaf boundary pixel to the central point was plotted at all angles and the curve was smoothed to determine the local maximum modal points. Figure 3(a) shows the results of leaf axial direction determination for a typical cabbage seedling. The algorithm was efficient for seedlings with less than five leaves. For seedlings with more than five leaves, error usually increases due to partly occluded leaves (Lin and Chang, 1999).

For vegetable seedlings of later growth stages, the estimation error of total leaf area usually increases as the extent of overlapped leaves increases. To solve this problem, we further developed an algorithm to determine the overlapped leaf area. The position and approximate shape of overlapped seedling leaves are initially located using elliptical Hough transform (Muammar and Nixon, 1991). In performing the Hough transform, the top-view image was processed with edge detection algorithm to find the pixels representing the boundary of leaves. The coordinates of these pixels were then transformed into a parameter space of all possible ellipses. The ellipses corresponding to individual leaves were then computed by matching the leaf boundary in several iterations until all leaves were matched. Figure 3(b) shows the results of leaf detection using the elliptical Hough transform for a typical top-view image of cabbage seedling. To determine the leaf area, leaf boundary in the overlapped region was approximated with the boundary of the corresponding ellipse. Individual leaf area and total leaf area can be estimated with the matched ellipses corresponding to individual leaves of the seedling (Chien and Lin, 2002).

To improve the accuracy of the leaf number estimation and leaf area measurement, we further incorporated two side-view images with the top-view image to extract and reconstruct the 3-

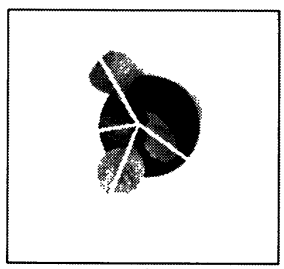

(a)

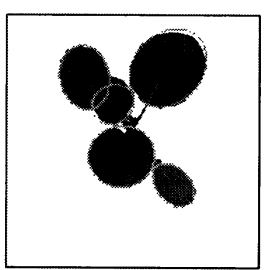

(b)

Fig. 3 Detection of seedling leaves with (a) local maximum modal point method, (b) elliptical Hough transform method. 


\section{GROWTH MEASUREMENT USING MACHINE VISION}

dimensional structure of selected vegetable seedlings, and thus to measure the seedling features. By registering correspondences between the three orthogonal images, the position, orientation and dimensions of leaves in a seedling can be more exactly derived than from single top-view image alone. The leaf skeletons traced in the side-view images can be used to correct the projected leaf area in the top-view image for better estimation of leaf area. The average relative errors of total leaf estimation for cabbage and broccoli seedlings were $14.5 \%$ and $13.1 \%$, respectively, using single top-view image. By incorporating orthogonal images, the relative errors significantly reduced to $1.6 \%$ and $4.9 \%$. Using this non-destructive measurement system, the continuous growth of vegetable seedlings can be effectively measured (Chien and Lin, 2005).

Compared with plant silhouette approach, the measurement process of the structural approach is relatively complicated. However, more seedling characteristics can be extracted depending on the development of specific image analysis algorithms.

\section{INTEGRATED SYSTEM FOR PLANT GROWTH MEASUREMENT AND MODELING}

The processes of plant feature measurement and structural modeling influence each other. The choice of features to be measured depends greatly on the purpose of plant model building. In recent years, plant growth modeling has emerged as an important area of interdisciplinary research. Developmental models of plant architecture can be constructed for a better understanding of plant development under various conditions (Jaeger and de Reffye, 1992; Room et al., 1994). To achieve realistic plant model with computer graphics, structural rules and measured parameters are both required.

\section{Plant growth functions}

A number of mathematical models have been developed to analyze and simulate plant growth (France and Thornley, 1984). In the model building process, a growth function is required to describe the measured data of plant growth. In our studies, simple mathematical relations based on logistic and Richards functions were used to describe the dynamic responses of selected vegetable seedlings (Charles-Edwards et al., 1986). The measured plant characteristics, plant height, total leaf area, top fresh weight and top dry weight, were fitted to the logistic function and Richards function using method of non-linear least squares. The model parameters were determined and comparisons were made for growth responses under various conditions. The prediction from the growth models allows us to compare the pertinent conditions for plant growth and thus better plant production management can be advised.

\section{Reconstruction of plant structure}

With the data from continuous measurements of a plant growing process, dynamic graphic simulation of plant growth can be achieved. We have developed a virtual plant system and the system was tested with a measurement experiment to determine the growth curves of pepper seedlings cultured in pots of different sizes to compare the effect of growth media quantity on the growth of various kinds of seedlings (Lin et al., 2001). The stereo vision measurements system combined with plant growth models, with the capability of computer graphic visualization, forms an integrated system for plant growth analyses that may be used in many areas both in fundamental research or practical plant production processes.

\section{CONCLUSIONS}

Two approaches of machine vision application for plant growth measurement are introduced and discussed in this paper. Estimation of plant dry weight, fresh weight, and total leaf area can be achieved using projection area from plant silhouette images. While this plant silhouette approach is relatively easy to be implemented, the estimation of plant features requires calibration 


\section{T. T. LIN ET AL.}

information from separate experiments. To directly measure plant leaf area, the problem of overlapped leaves needs to be overcome. With the stereo vision approach, the hidden leaf boundary can be reconstructed and the total leaf area can be calculated without predetermined calibration relationship. Three-dimensional information of plant features and structure can be determined with the aid from a second camera. The data determined from the machine vision system can be fed into a plant structure model based on plant growth functions for 3-dimensional computer graphic simulation. The stereo machine vision system combined with the plant structure model provides information not only from directly measurable features by image analysis but also predicted characteristics from simulation.

\section{REFERENCES}

Bailey, W. G., Steward, R. B. 1982. A method for assessing leaf area. Can. J. Plant Sci. 62: 211-214.

Charles-Edwards, D. A., Doley, D., Rimmington, G. M. 1986. Modelling Plant Growth and Development. Academic Press, Sydney, pp 235.

Chien, C. F., Lin, T. T. 2002. Leaf area measurement of selected vegetable seedlings using elliptical Hough transform. Trans. ASAE 45: 1669-1677.

Chien, C. F., Lin, T. T. 2005. Non-destructive growth measurement of selected vegetable seedlings using orthogonal images. Trans. ASAE 48: 1953-1961.

Daughtry, C. 1990. Direct measurement of canopy structure. Remote Sens. Rev. 5: 45-60.

Fournier, C., Andrieu, B. 1998. A 3D architectural and process-based model of maize development. Ann. Bot. 81: 233-250.

France, J., Thornley, J. H. M. 1984. Mathematical Models in Agriculture: A Quantitative Approach to Problems in Agriculture and Related Sciences. Butterworth, London.

Gonzales, R. C., Woods, R. E. 2002. Digital Image Processing, Ed. 2. Prentice Hall, Upper Saddle River, New Jersey.

Hack, G. R. 1989. On-line measurement of plant growth in the greenhouse. Acta Hortic. 248: 337-343.

Henten, E. J. V., Bontsema, J. 1995. Non-destructive crop measurement by image processing for crop growth control. J. Agric. Eng. Res. 61: 97-105.

Howarth, M. S., Stanwood, P. C. 1993. Measurement of seedling growth rate by machine vision. Trans. ASAE 36: 959-963.

Ivanov, N., Boissard, P., Chapron, M., Andrieu, B. 1995. Computer stereo plotting for 3-D reconstruction of a maize canopy. Agric. For. Meterol. 75: 85-102.

Jaeger, M., de Reffye, P. H. 1992. Basic concepts of computer simulation of plant growth. J. Biosci. 17: 275-291.

Lin, T. T., Cheng, S. F., Lin, T. H., Tsai, M. R. 1998. Non-destructive growth measurement of selected vegetable seedlings using machine vision. Proc. Inst. Biol. Eng. 1: B38-B46.

Lin, T. T., Chang, J. M. 1999. Vegetable seedling feature extraction using stereo imaging. ASAE Paper No. 993007. St. Joseph, MI: ASAE.

Lin, T. T., Liao, W. C., Chien, C. F. 2001. 3D graphical modeling of vegetable seedlings based on a stereo machine vision system. ASAE Paper No. 013137. St. Joseph, MI: ASAE.

Meyer, G. E., Davison, D. A. 1987. An electronic image plant growth measurement system. Trans. ASAE 30: $242-247$.

Muammar, H. K., Nixon, M. 1991. Tristage Hough transform for multiple ellipse extraction. IEEE Proc. 138: 27-35.

Nyakwende, E., Paull, C. J., Atherton, J. G. 1997. Non-destructive determination of leaf area in tomato plants using image processing. J. Hortic. Sci. 72: 255-262.

Palit, P., Battacharyya, A. C. 1984. Measurement of leaf area per plant of white jute (Corchorus capsularis L.) and tossa jute (Corchorus olitorious L.) using the average specific leaf weight value. Trop. Agric. 61: 5962,80 .

Prusinkiewicz, P. W., Remphrey, W. R., Davidson, C. G., Hammel, M. S. 1994. Modeling the architecture of expanding Fraxinus pennsylvanica shoots using L-systems. Can. J. Bot. 72: 701-714. 


\section{GROWTH MEASUREMENT USING MACHINE VISION}

Robinson, P. P., Shands, W. A., Stormann, C. L., Simpson, G. W. 1970. An improved optical planimeter for measuring the area of detached leaves used in estimating populations of aphids. J. Econ. Ent. 63: 11591162.

Room, P. M., Maillette, L., Hanan, J. S. 1994. Module and metamer dynamics and virtual plants. Adv. Ecol. Res. 25: 105-157.

Shibata, T., Iwao, K., Takano, T. 1992. Growth prediction of lettuce plants by image processing. Acta Hortic. 319: 689-694.

Shimizu, H., Heins, R. D. 1995. Computer-vision-based system for plant growth analysis. Trans. ASAE 38: 959-964.

Singh, H. P., Singh, R. V. 1983. Methods of leaf area estimation of soybean from linear measurement. Legume Res. 6: 18-20.

Storlie, C. A., Stepanek, A., Meyer, G. E. 1989. Growth analysis of whole plants using video imagery. Trans. ASAE 32: 2185-2189.

Suh, S. R., Miles, G. E. 1988. Measurement of morphological properties of tree seedlings using machine vision and image processing. ASAE Paper No. 88-1542. St. Joseph, MI: ASAE.

Suzuki, T. 1995. Measurement of growth of plug seedlings by image processing in broccoli. Acta Hortic. 399: $263-267$.

Tsai, W. H. 1985. Moment-preserving thresholding: a new approach. Comput. Vis., Graph. Image Process. 29: $377-393$.

Trooien, T. P., Heermann, D. F. 1992. Measurement and simulation of potato leaf area using image processing III. Measurement. Trans. ASAE 35: 1719-1721.

Wolfe, R. R., Sandler, W. E. 1985. An algorithm for stem detection using digital image analysis. Trans. ASAE 28: 641-644.

Zhang, T. Y., Suen, C. Y. 1984. A fast parallel algorithm for thinning digital pattern. Comm. ACM 27: 236239. 